\title{
Severe Acquired Primary Hypothyroidism in Children and its Influence on Growth: A Retrospective Analysis of 43 Cases
}

\author{
Authors \\ Marianne Becker ${ }^{1}$, Oliver Blankenstein², Erwin Lankes', Dirk Schnabel'1, Heiko Krude²
}

Affiliations

1 Sozial-Pädiatrisches Zentrum, Charité Universitätsmedizin Berlin, Berlin, Germany

2 Institute for Experimental Pediatric Endocrinology, Charité Universitätsmedizin Berlin, Berlin, Germany

\section{Key words}

thyroiditis, catch-up growth, pubertal state, presenting symptoms

\section{received 16.03.2021 \\ revised $\quad 07.06 .2021$ \\ accepted 23.06.2021}

published online $\quad 04.10 .2021$

\section{Bibliography}

Exp Clin Endocrinol Diabetes 2022; 130: 217-222

DOI 10.1055/a-1538-8241

ISSN 0947-7349

(C) 2021. Thieme. All rights reserved.

Georg Thieme Verlag, Rüdigerstraße 14,

70469 Stuttgart, Germany

\author{
Correspondence \\ Dr. Marianne Becker \\ Department of Pediatric Endocrinology and Diabetology \\ (DECCP), Kannerklinik, Centre Hospitalier de Luxembourg \\ 4. rue Barblé \\ 1210 Luxembourg \\ Luxembourg \\ Tel.: + 0035244118733 , Fax: + 0035244116466 \\ becker.marianne@chl.lu
}

\begin{abstract}
Introduction Severe acquired hypothyroidism in childhood is a rare condition, mostly caused by autoimmune thyroiditis. Scarce and inconsistent data based on small patient numbers exist concerning its impact on growth in height.

Methods Patient files at a single centre university hospital over 8 years were retrospectively reviewed. We identified 43 patients (mean age 10.6 years, 3.3-15.25, $59 \%$ prepubertal, $88 \%$ females) in a cohort of children older than 3 years with an initial TSH $>30 \mathrm{mlU} / \mathrm{l}$ and reduced T4 or fT4; congenital and drug-induced hypothyroidism were excluded.

Results All patients had signs of autoimmune thyroiditis (93\% positive autoantibodies, $95 \%$ typical ultrasonography, $63 \%$ goiter). Median TSH was $100 \mathrm{mlU} / \mathrm{l}[0.3-4 \mathrm{mIU} / \mathrm{I}])$, median fT4 $3.55 \mathrm{pg} / \mathrm{ml}$ [8-19 pg/ml], median T4 $2.85 \mu \mathrm{g} / \mathrm{dl}[5.3-11 \mu \mathrm{g} / \mathrm{dl}]$. Presenting symptoms included goiter (26\%), tiredness (23\%), weight gain (19\%), and growth retardation (19\%). The diagnosis was made incidentally in $26 \%$ patients. In $75 \%$ growth was retarded (median height standard deviation score (SDS)-0.55), in $17 \%$ height SDS was $<-2$ at diagnosis. Midparental height SDS at diagnosis correlated significantly with T4 and fT4 $(r=0.77, p=0.0012$ and $r=0.53, p=0.021$ respectively). Catchup growth under T4 substitution was significantly greater in prepubertal than in pubertal children ( $p$ 0.049).

Conclusion This so far largest pediatric cohort with severe acquired hypothyroidism confirms a serious impact on growth which, however in most cases, showed a certain catch-up growth after adequate L-thyroxine therapy. The pubertal state seems to be important for catch-up growth. A significant number of patients were not diagnosed clinically, although affected by severe hypothyroidism.
\end{abstract}

\section{Introduction}

Hypothyroidism in childhood may be either congenital or acquired. Epidemiological studies to estimate the prevalence of hypothyroidism in childhood are very rare. In one population-based study in Scotland in 0-22 years old individuals, acquired hypothyroidism was found to be more frequent with a prevalence of 1 in 1450 , compared to congenital hypothyroidism with a prevalence of 1 in 3700 [1]. The etiology of primary acquired hypothyroidism mainly includes autoimmune thyroiditis, i. e. Hashimoto's disease, with additional iatrogenic cases occurring in inappropriate L-thyroxine (LT4)-substitution after thyroidectomy (either surgical or by radioiodine treatment) or as a complication of drug treatment in the setting of Graves' disease. Hypothyroidism caused by iodine deficiency in children in western countries has become very rare, but can still occur in families that follow a restricted diet [2].

As the most frequent cause of acquired hypothyroidism, Hashimoto's thyroiditis occurs almost exclusively after the age of 3 years [3] and is by far more frequent in girls than in boys. Several studies have focused on the prevalence and course of Hashimoto's thyroiditis in childhood [4-13]. However, in most patients having a typical ultrasound aspect and positive thyroglobulin ( $\mathrm{Tg}$ ) and/or thyroid peroxidase (TPO) antibodies, no manifest hypothyroidism is observed; if thyroid function is altered, an isolated increase of TSH with normal T4 has been found in most cases [14]. 
The lack of thyroid hormone has detrimental consequences on the growth of the child as outlined in severely ill cases of historic untreated congenital hypothyroidism. However, the acquired lack of thyroid hormone can also significantly impair further development. If hypothyroidism occurs within the first two years of life, cognitive development can be compromised [3]. Later, mainly somatic growth is delayed with the hazard of short stature as final height. A precise characterization of pediatric patients with acquired hypothyroidism especially concerning its impact on their further development is lacking. Only a few studies have focused on the influence of this condition on growth, with contradicting results [15-19].

In the light of scarce data about pediatric patients with manifest acquired hypothyroidism, we investigated another cohort of patients with acquired hypothyroidism at a pediatric single center to evaluate the diagnostic findings, describe the study cohort, and evaluate the influence of the temporary lack of thyroid hormone on growth.

\section{Materials and Methods}

We retrospectively evaluated all available thyroid function tests in a patient cohort at a tertiary pediatric university center from 1.1 .2004 to 31.12 .2012 . To identify patients with acquired primary hypothyroidism and therefore to avoid overlap with congenital hypothyroidism, we included only patients who were older than 3 years, because acquired hypothyroidism occurs almost exclusively after the age of 3 years $[3,20]$.

The study was approved by the Ethics Committee of the Charité in Berlin, Germany.

To include only patients with manifest hypothyroidism, we selected patients with an initial TSH $>30 \mathrm{mIU} / \mathrm{I}$ (reference value $0.3-4$ $\mathrm{mIU} / \mathrm{l})$ and reduced T4 and/or fT $4(<6 \mu \mathrm{g} / \mathrm{dl}$ and $<8 \mathrm{pg} / \mathrm{ml}$, respectively). We did not analyze hypothyroid patients with complications of treatment - like insufficient substitution in congenital or post thyroidectomy hypothyroidism or overtreatment in Graves' disease.

We traced the patients' clinical data and their respective charts to acquire information on thyroid and anthropometric parameters. We estimated the volume of thyroid glands according to the sonographic criteria of Liesenkötter [21].

T4, fT4, TSH as well as TPO and Tg antibodies were measured by electrochemiluminescence immunoassays (Elecsys, Roche Diagnostics, Mannheim, Germany) at the Labor Berlin.

Treatment consisted of LT4 and was adapted according to regular fT 4 and TSH measurements (initially after 2 and 4 weeks and in the follow-up every 3-6 months).

Physical examinations were conducted for height, weight, and pubertal stage, which were documented according to Tanner and testicular volume. Children were classified as prepubertal if girls had Tanner stage 1 and if boys had a testicular volume of $\leq 3 \mathrm{ml}$.

To evaluate growth, we calculated the height standard deviation score (SDS) based on Reinken et al. [22]. Mid-parental height (MPH) was calculated based on the documented height of both parents. MPH data was available for 30 patients. The MPH SDS was calculated using the following formula: (MPH-normal adult height/SDS) .

The value of height SDS minus MPH SDS indicates whether the child is growing as per his/her genetic target height. A negative (height SDS-MPH SDS) indicates that the child is shorter than expected, while a positive (height SDS-MPH SDS) indicates that the child is taller than expected for his genetic target height. To evaluate the magnitude of catch-up growth after initiating the thyroxine replacement therapy, the difference between the (height SDSMPH SDS) value at diagnosis and the last available value was calculated. In addition, the individual growth charts were assessed for change in the growth percentiles.

Growth retardation was diagnosed if height SDS (or height SDSMPH SDS) before diagnosis was greater (>0.5 SDS) than height SDS (or height SDS-MPH SDS) at manifestation and/or if height SDS (or height SDS-MPH SDS) at diagnosis was less (<0.5 SDS) than last documented height SDS (or last documented height SDS-MPH SDS). Growth retardation was also assumed if height SDS-MPH SDS at diagnosis was <-1 (not applicable for syndromic patients).

Statistical analyses were performed using SPSS and R-Statistical Software. We performed Spearman Rank correlation and a MannWhitney U-test.

\section{Results}

We screened all the patient files at our center between 2004 and 2013 and identified 99 patients who were older than 3 years and had a TSH $>30 \mathrm{mIU} / \mathrm{l}, \mathrm{T} 4<6 \mu \mathrm{g} / \mathrm{dl}$, and/or fT $4<8 \mathrm{pg} / \mathrm{ml}$. Fifty-six of these patients were either suffering from congenital hypothyroidism, or hypothyroidism was drug-induced or occurred after thyroidectomy.

The remaining 43 patients were considered to be affected by acquired primary manifest hypothyroidism and were investigated further. Of these 38 were female, so the male to female ratio was $1: 8$. The mean age at diagnosis was 10.6 years and $59 \%$ were prepubertal (shown in $>$ Fig. 1a).

Most of the patients underwent thyroid function tests for goiter, tiredness, growth retardation, and weight gain. Other reasons included constipation, cold hand and feet, and joint pain. Remarkably, in 11 patients (26\%) thyroid function test was performed in the setting of a routine blood test and hypothyroidism was therefore diagnosed incidentally (see $>$ Fig. 1b). In two patients, Down syndrome and in one patient Tuner syndrome had been previously diagnosed.

Median TSH was $101 \mathrm{mlU} / \mathrm{l}$, median $\mathrm{fT} 4$ was $3.55 \mathrm{pg} / \mathrm{ml}$, and median T4 was $2.85 \mu \mathrm{g} / \mathrm{dl}$. A continuum of severity of hypothyroidism was observed to have significant negative correlation of TSH and fT4 or T4 (fT4: $r=-0.55, p=0.0016 ; T 4: r=-0.82, p=0.000006$ ) (see - Fig. 2). Seven of the 11 patients, who were diagnosed incidentally without clinical symptoms, were found with a milder hypothyroidism $(\mathrm{T} 4>5 \mu \mathrm{g} / \mathrm{dl}$ or free $\mathrm{T} 4>5 \mathrm{pg} / \mathrm{ml}$ ), but four of these patients were affected by severe hypothyroidism $(\mathrm{T} 4<3.5 \mu \mathrm{g} / \mathrm{dl}$ or free $\mathrm{T} 4<1.5 \mathrm{pg} / \mathrm{ml}$ ).

In 41 patients, thyroid ultrasonography was performed at diagnosis. In 40 of 41 patients, the ultrasonography showed typical signs of thyroiditis (heterogeneous echotexture and hypervascularization). Sixty-six percent of the participents had goiter, $32 \%$ had a normal seized thyroid, and $2 \%$ had a hypoplastic thyroid (see Table 1). Ninety-three percent of the participants were positive for TPO antibodies and $70 \%$ tested positive for TG antibodies. Two patients did not have elevation of any of these two antibodies but 

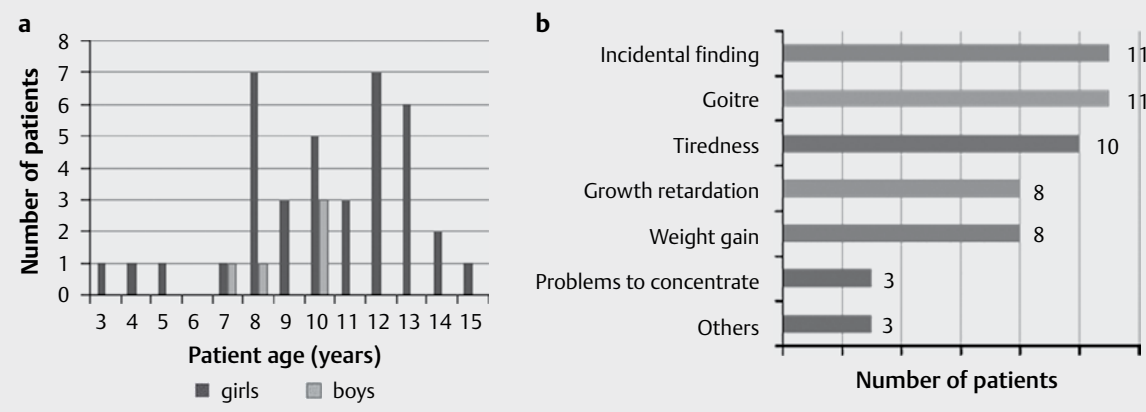

- Fig. 1 a Age distribution of the cohort with severe primary hypothyroidism. b Presenting symptoms that led to the prescription of a thyroid function test.

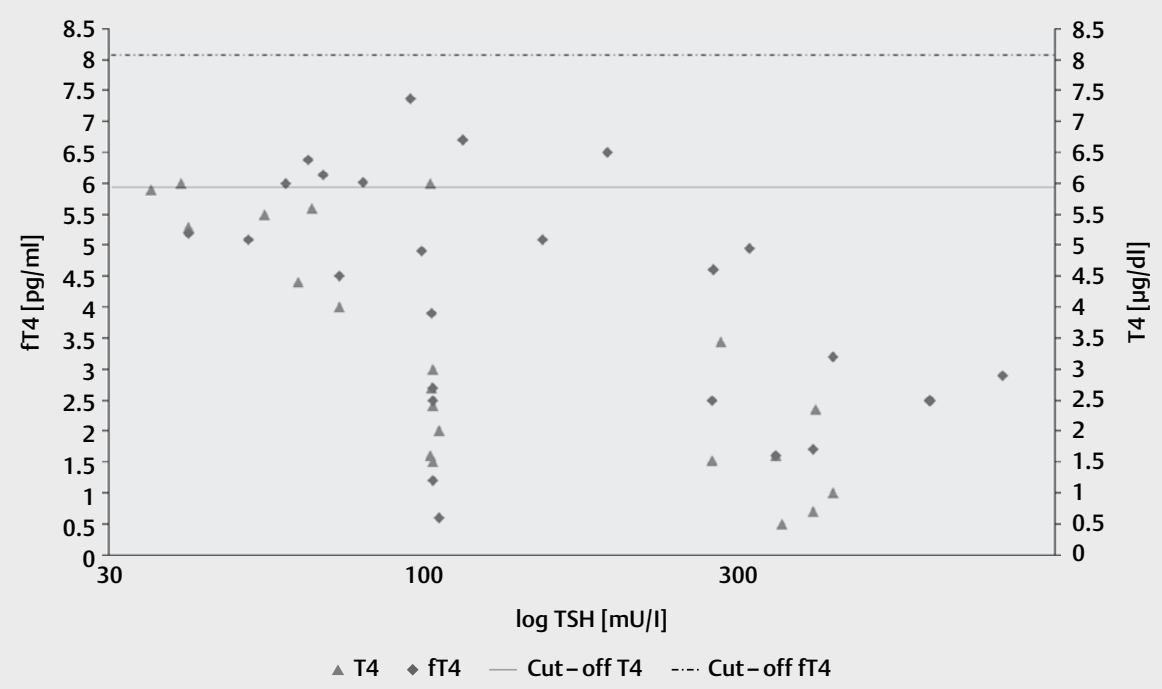

- Fig. 2 This figure illustrates the severity of hypothyroidism in our cohort. It shows the correlation between TSH [mIU/ml] and T4 $[\mu \mathrm{g} / \mathrm{dl}]$ or fT4 $[\mathrm{pg} / \mathrm{ml}]$. Values of T4 are shown as $\mathbf{\Lambda}$, and that of fT4 as a $\bullet$. The cut-off level for T4 is indicated as a '.......' line, and the cut-off level for fT4 is indicated as '_-_-_' line.

showed signs of antibody-negative Hashimoto's thyroiditis at thyroid ultrasonography. Thus, all 43 patients who were diagnosed by their laboratory values to have acquired manifest hypothyroidism were affected by Hashimoto's thyroiditis (see - Table 1).

Growth data were available for 42 patients. To estimate the impact of hypothyroidism on growth, each individual's height at diagnosis was evaluated as "height-SDS" (to identify the deviation from normal age and gender matched reference height) (shown in - Fig. 3a). At diagnosis, $75 \%$ of patients had a negative height-SDS, suggesting a growth deficit of the cohort compared with an ageand gender-matched reference cohort, while five patients had a manifest non-syndromic short stature as they had a height SDS <-2 (see $>$ Fig. 3a).

In addition, 20 patients (47\%) were classified as having growth retardation at diagnosis based on their growth charts. In total, only 13 patients had no growth retardation (30\%). In 10 patients,
- Table 1 The diagnostic findings in our study cohort

\begin{tabular}{|l|l|}
\hline Finding & $\begin{array}{l}\text { Patients [\%] } \\
\text { (number) }\end{array}$ \\
\hline Ultrasonography: thyroid size & \\
\hline Goiter & $66(27)$ \\
\hline Normal size & $32(13)$ \\
\hline Hypoplastic & $2(1)$ \\
\hline $\begin{array}{l}\text { Ultrasonography (41): typical signs for } \\
\text { thyroiditis }\end{array}$ & $98(40)$ \\
\hline Anti-TPO-ab positive & $93(40)$ \\
\hline Anti-TG-ab positive & $70(30)$ \\
\hline No Ab positive & $5(2)$ \\
\hline $\begin{array}{l}\text { No Ab positive and no typical signs at } \\
\text { ultrasonography }\end{array}$ & $0(0)$ \\
\hline TPO: thyrid peroxidase, ab: antibody, TG: thyroglin
\end{tabular}

TPO: thyroid peroxidase, ab: antibody, TG: thyroglobulin 

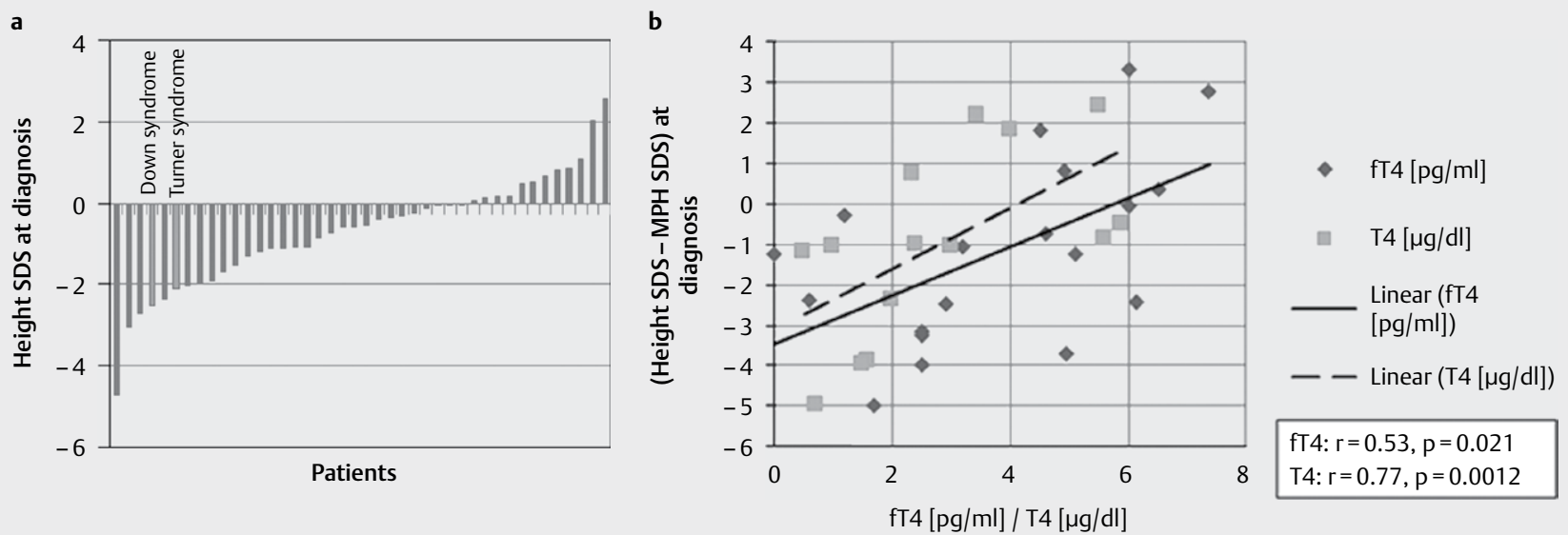

- Fig. 3 a Height-standard deviation score (SDS) at diagnosis. b Correlation between midparental height standard deviation score (MPH SDS) at diagnosis and T4 [ $\mathrm{\mu g} / \mathrm{dl}] / \mathrm{fT} 4[\mathrm{pg} / \mathrm{ml}]$. Values of T4 are shown as and values of fT4 as a $\bullet$. Correlation between MPH SDS at diagnosis and T4 is shown as a '____' line, and that between MPH SDS at diagnosis and fT4 as a '__' line, ' $r$ ' denotes Spearman's rank correlation coefficient.

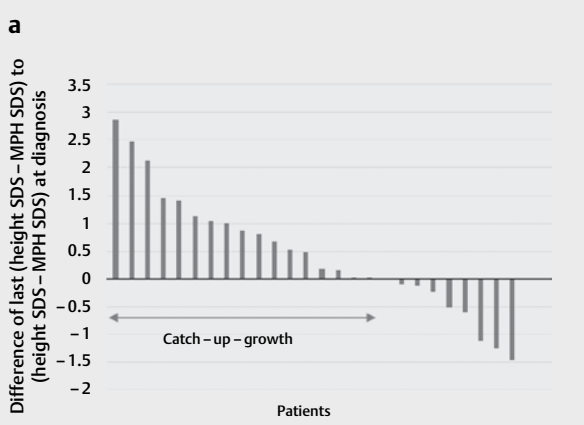

Patients
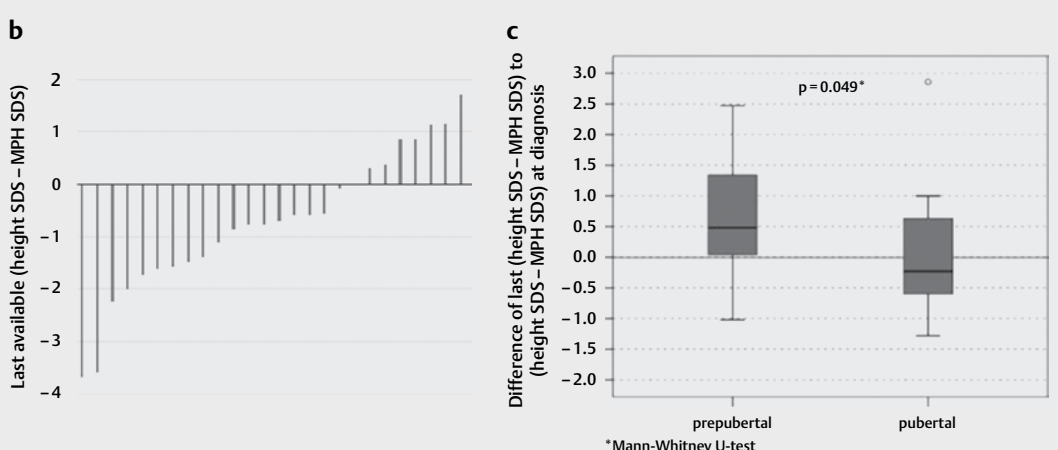

- Fig. 4 a Difference of midparental height standard deviation score (MPH SDS) at diagnosis to last available MPH SDS, illustrating a catch-up growth in most patients. $\mathbf{b}$ Last available midparental height standard deviation score (MPH SDS). $\mathbf{c}$ Catch-up growth under L-thyroxine treatment (difference of last available MPH SDS and MPH SDS at diagnosis): prepubertal versus pubertal children. Statistical analysis was performed using the Man-Whitney-U-test.

growth retardation could not be evaluated because less than two values of their growth were available $<6$ months apart from each other after manifestation, or neither MPH nor the height values before diagnosis were available. Two syndromic patients were excluded from the analysis. One Down syndrome patient was classified as having growth retardation, as her growth before developing hypothyroidism was better ( -1.98 SDS) than at diagnosis (-2.5 SDS) and she showed an incomplete catch-up growth under treatment (last available height:-2.17 SDS).

To evaluate the impact of the severity of hypothyroidism on growth, we calculated the correlation of $\mathrm{fT} 4$ or T4 with the MPH SDS at diagnosis. We found a significant correlation between the fT4 or T4 values at diagnosis and MPH SDS at diagnosis (fT4: $r=0.53, p=0.021 ; T 4: r=0.77, p=0.0012$ ) (see $>$ Fig. $3 b$ ).

We could evaluate the follow-up of 33 patients after a median follow-up time of 3.4 years ( $0.6-10.3$ years). Besides, $58 \%$ of the initially prepubertal patients were followed at least until the age of
12 years. MPH was available for 26 of these 33 patients. To evaluate the magnitude of catch-up growth, we calculated the difference (height SDS-MPH SDS) at diagnosis and at their last visit (delta height SDS-MPH SDS). Complete catch-up growth was defined as a difference of $<0.5$ SDS to the value before hypothyroidism or, if the value before hypothyroidism was not available, as the last documented (height SDS-MPH SDS) value of <-1 SDS. Forty percent of children achieved a complete catch-up growth under treatment. Most children experienced some catch-up growth (positive delta height SDS-MPH SDS) (shown in > Fig. 4a), but the majority (18 out of 26 patients) still had a negative (height SDS-MPH SDS) value at their last documented visit (see $>$ Fig. 4b). Catch-up growth was significantly greater in prepubertal (median delta (height SDS-MPH SDS) + 0.5) than in pubertal children (median delta (height SDSMPH SDS)-0.2) (p 0.049) (see > Fig. 4c). 


\section{Discussion and Conclusions}

This study evaluated severe acquired hypothyroidism in the so-far largest cohort of children. In line with many other studies, all children in our study population were affected by Hashimoto's thyroiditis. However, severe acquired hypothyroidism was rare because over a period of 8 years only 43 children were diagnosed.

The sex ratio (female: male) of pediatric patients affected by Hashimoto's thyroiditis has been reported to be in the range of 2:1 [23] to 6.5:1 [4]. A higher female preponderance was observed in the adult population, with a female to male ratio of 8:1[24]. Likewise, in our pediatric population with Hashimoto's thyroiditis who were affected by severe hypothyroidism, we observed a female to male ratio of $8: 1$, similar to that described for adult patients. Thus, it can be inferred that females have a greater tendency to develop Hashimoto's disease, particularly of a more severe form not only in adulthood but also in childhood.

In general, the age of manifestation of Hashimoto's thyroiditis has been described more in the pubertal than in the prepubertal range [13]. Interestingly most of our hypothyroid patients were prepubertal, although, their age peak was close to puberty. This is in line with two particular studies of 19 and 26 children with hypothyroidism and Hashimoto's thyroiditis respectively, that pointed out a higher prevalence of overt hypothyroidism in the setting of Hashimoto's thyroiditis in prepubertal children [12, 25]. In summary, these data suggest that the natural course of Hashimoto's thyroiditis is more aggressive in the prepubertal age group.

Although we included only patients with severe hypothyroidism, a quarter of our patients were diagnosed incidentally without any specific clinical sign of hypothyroidism resulting in the prescription of a thyroid function test. While two other studies by Skarpa et al. and Vries et al. $[12,17]$ also observed that most patients with Hashimoto's thyroiditis were asymptomatic, these study populations included children who were either hypothyroid, euthyroid, or hyperthyroid [17]. To our knowledge, this is the only study to have included exclusively hypothyroid patients with Hashimoto's thyroiditis, with a remarkably high number of patients who were diagnosed incidentally.

Concerning thyroid findings, ultrasonography was more sensitive in diagnosing Hashimoto's thyroiditis than antibodies while TG antibodies were less frequently elevated compared to TPO antibodies in these patients, consistent with the findings of other studies $[5,9,12]$.

Although there was a shift to negative height SDS values at diagnosis in our cohort (29/42 patients), only five patients had a nonsyndromic short stature (height SDS <-2.0). The severity of the growth retardation was significantly correlated with the severity of hypothyroidism at diagnosis. The correlation was stronger with T4 versus fT4 measurements (see $>$ Fig. $\mathbf{3 b}$ ). This is most likely due to the greater robustness of total T4 values in this particular cohort of children with thyroid auto-immune antibodies that possibly interfere with serum thyroid hormone measurements. Therefore, our data clearly confirm the negative impact of manifest hypothyroidism on growth. However, we could also show that most patients experienced catch-up growth (positive delta height SDS-MPH zscore) under adequate LT4 substitution with complete catch-up growth in nearly half of the children. At the end of follow-up, the majority of patients still had a negative (height SDS-MPH SDS) value, but the height of only two children remained below SDS of-2. This suggests that a sufficient treatment with LT4 prevents short stature in the vast majority of children but cannot completely compensate for minor height losses. The irreversibility of height loss is in line with the findings of Pantsiotou and Rivkees, who studied 29 and 24 children, respectively, with juvenile primary hypothyroidism and showed a permanent final height deficit $[15,16]$. In contrast to the final height loss described by Rivkees, our patients were less short: in our cohort, two patients had a height SDS $<-2$ at their last visit while all patients of Rivkees' cohort had a final height of $<-2$ SDS. This discrepancy might be explained by the fact that Rivkees included only patients with longstanding hypothyroidism who were all very short at the time of diagnosis.

Our data shows significantly better catch-up growth in prepubertal versus pubertal children and is in line with the studies of Ranke (20 prepubertal children with primary hypothyroidism) [19] and Chiesa (26 prepubertal and six pubertal children)[18], who demonstrated a complete catch-up growth in prepubertal $[18,19]$, but not in pubertal children [18].

The growth data of our study contradict those of de Vries et al. who reported a significantly lower height SDS at diagnosis in hypothyroid children with Hashimoto's thyroiditis compared to a euthyroid Hashimoto's thyroiditis subgroup, while this difference diminished during follow up under treatment with LT4 [17].

In conclusion, this is the first study to provide the largest cohort of children with Hashimoto's thyroiditis and severe acquired hypothyroidism and the first to primarily focus on this rare hypothyroid subgroup. Growth retardation was observed in the majority of patients at diagnosis and correlated significantly with the severity of hypothyroidism. However, adequate treatment with LT4 resulted in the prevention of short stature in most patients-except for two patients-although in more than $50 \%$ of the patients a final height reduction remained when considering $\mathrm{MPH}$ values and longitudinal changes of their growth. Remarkably, a quarter of these severely hypothyroid patients were discovered incidentally by random blood tests. It might therefore be reasonable to consider a thyroid function test in every child with a decline in growth rate to avoid the development of more severe hypothyroidism and hence the risk of an irreversible impact on growth.

\section{Acknowledgment}

We are grateful to Sean Sacariu, Ph.D., who critically revised the manuscript.

\section{References}

[1] Hunter I, Greene SA, MacDonald TM et al. Prevalence and aetiology of hypothyroidism in the young. Arch Dis Child 2000; 83: 207-210

[2] Booms S, Hill E, Kulhanek L et al. lodine deficiency and hypothyroidism from voluntary diet restrictions in the US: case reports. Pediatrics 2016; 137: doi:10.1542/peds.2015-4003

[3] Lafranchi S. Thyroiditis and acquired hypothyroidism. Pediatr Ann 1992; 21: 32-39 
[4] Inoue M, Taketani N, Sato T et al. High incidence of chronic lymphocytic thyroiditis in apparently healthy school children: epidemiological and clinical study. Endocrinol Jpn 1975; 22: 483-488

[5] Roth C, Scortea M, Stubbe P et al. Autoimmune thyreoiditis in childhood--epidemiology, clinical and laboratory findings in 61 patients. Exp Clin Endocrinol Diabetes 1997; 105: 66-69. doi:10.1055/s-0029-1211937

[6] Marwaha RK, Tandon N, Karak AK et al. Hashimoto's thyroiditis: Countrywide screening of goitrous healthy young girls in postiodization phase in India. J Clin Endocrinol Metab 2000; 85: 3798-3802. doi:10.1210/jcem.85.10.6924

[7] Dötsch J, Hau M, Heidemann P et al. Verlauf, therapie und komorbidität bei Hashimoto-thyreoiditis im kindesalter. Monatsschr Kinderheilkd 2003; 151: 528-531. doi:10.1007/s00112-002-0657-y

[8] Zak T, Noczyńska A, Wasikowa R et al. Chronic autoimmune thyroid disease in children and adolescents in the years 1999-2004 in Lower Silesia, Poland. Horm Athens Greece 2005; 4: 45-48

[9] Gopalakrishnan S, Chugh PK, Chhillar M et al. Goitrous autoimmune thyroiditis in a pediatric population: a longitudinal study. Pediatrics 2008; 122: e670-e674. doi:10.1542/peds.2008-0493

[10] McGrogan A, Seaman HE, Wright JW et al. The incidence of autoimmune thyroid disease: a systematic review of the literature. Clin Endocrinol (Oxf) 2008; 69: 687-696. doi:10.1111/j.1365-2265.2008.03338.x

[11] Demirbilek H, Kandemir N, Gonc EN et al. Assessment of thyroid function during the long course of Hashimoto's thyroiditis in children and adolescents. Clin Endocrinol (Oxf) 2009; 71: 451-454. doi:10.1111/j.1365-2265.2008.03501.x

[12] Skarpa V, Kousta E, Tertipi A et al. Epidemiological characteristics of children with autoimmune thyroid disease. Horm Athens Greece 2011; 10: 207-214

[13] Wasniewska M, Corrias A, Salerno M et al. Thyroid function patterns at Hashimoto's thyroiditis presentation in childhood and adolescence are mainly conditioned by patients' age. Horm Res Paediatr 2012; 78: 232-236. doi:10.1159/000343815

[14] Aversa T, Corrias A, Salerno M et al. Five-year prospective evaluation of thyroid function test evolution in children with Hashimoto's thyroiditis presenting with either euthyroidism or subclinical hypothyroidism. Thyroid Off J Am Thyroid Assoc 2016; 26: 1450-1456. doi:10.1089/ thy. 2016.0080
[15] Rivkees SA, Bode HH, Crawford JD. Long-term growth in juvenile acquired hypothyroidism: the failure to achieve normal adult stature. N Engl J Med 1988; 318: 599-602. doi:10.1056/ NEJM198803103181003

[16] Pantsiouou S, Stanhope R, Uruena M et al. Growth prognosis and growth after menarche in primary hypothyroidism. Arch Dis Child 1991; 66: 838-840

[17] de Vries L, Bulvik S, Phillip M. Chronic autoimmune thyroiditis in children and adolescents: at presentation and during long-term follow-up. Arch Dis Child 2009; 94: 33-37. doi:10.1136/ adc. 2007.134841

[18] Chiesa A, Gruñeiro de Papendieck L, Keselman A et al. Final height in long-term primary hypothyroid children. J Pediatr Endocrinol Metab JPEM 1998; 11: 51-58. doi:10.1515/jpem.1998.11.1.51

[19] Ranke MB, Schwarze CP, Mohnike K et al. Catch-up growth after childhood-onset substitution in primary hypothyroidism: is it a guide towards optimal growth hormone treatment in idiopathic growth hormone deficiency? Horm Res 1998; 50: 264-270. doi: $10.1159 / 000023288$

[20] Foley TP, Abbassi V, Copeland KC et al. Brief report: hypothyroidism caused by chronic autoimmune thyroiditis in very young infants. $\mathrm{N}$ Engl J Med 1994; 330: 466-468. doi:10.1056/NEJM199402173300704

[21] Liesenkötter KP, Kiebler A, Stach B et al. Small thyroid volumes and normal iodine excretion in Berlin schoolchildren indicate full normalization of iodine supply. Exp Clin Endocrinol Diabetes 1997; 105: 46-50. doi:10.1055/s-0029-1211932

[22] Reinken L, van Oost G. Longitudinal physical development of healthy children 0 to 18 years of age. Body length/height, body weight and growth velocity. Klin Padiatr 1992; 204: 129-133. doi:10.1055/s-2007-1025337

[23] Rallison ML, Dobyns BM, Keating FR et al. Occurrence and natural history of chronic lymphocytic thyroiditis in childhood. J Pediatr 1975; 86: 675-682

[24] Beeson PB. Age and sex associations of 40 autoimmune diseases. Am J Med 1994; 96: 457-462

[25] Kucharska AM, Witkowska-Sẹdek E, Labochka D et al. Clinical and biochemical characteristics of severe hypothyroidism due to autoimmune thyroiditis in children. Front Endocrinol 2020; 11: doi:10.3389/fendo.2020.00364 